The mechanism of the ejection itself has been described by Dr Kiepenheuer. The observations demonstrate that the following emissions take place, either from the flare or from neighbouring regions:

I. Ultra-violet and X-radiation, producing the ionospheric disturbances, some in layers at about $90 \mathrm{~km}$. and others about $75 \mathrm{~km}$.

2. Corpuscules with a speed of the order of $500-1000 \mathrm{~km}$. $/ \mathrm{sec}$., producing the magnetic storms and the aurorae.

3. Corpuscules with a speed of $50,000 \mathrm{~km} . / \mathrm{sec}$, of which no special terrestrial effects are observed.

4. Cosmic radiation.

During the passage of the corpuscules through the chromosphere and corona, electromagnetic radio-waves are generated, which at each height have the frequency of the corresponding plasma oscillation and appear in the order of increasing wave-lengths. Associated with the passage of the $500 \mathrm{~km}$. corpuscules are outbursts of type II; associated with the $50,000 \mathrm{~km}$. corpuscules are outbursts of type III.

It remains to investigate in how far these streams find their origin in the following individual objects:

(a) Surges, which are found to ascend and to descend along the same path, either when they are observed at the limb with the polarizing filter or when they are seen projected against the solar disk by their Doppler effects;

(b) Already existing quiescent prominences, which are activated and ejected by the influence of the flare, and which often reappear at the same place.

In both cases it seems as if a part of the material was ejected while another part is falling back.

There is a difference of opinion concerning the origin of the cosmic rays. Wild assumes that they are the $50,000 \mathrm{~km}$. corpuscules, subsequently accelerated. Alfvén thinks that they are formed in the corona by magneto-hydrodynamic shock waves, proceeding from the flare.

\title{
DISCUSSION
}

H. Alfvén: I do not think that Wild's type IIr bursts with velocities of $50,000 \mathrm{~km}$. $/ \mathrm{sec}$. are associated with cosmic rays, as implied in other speakers' remarks.

M. MinnaERT: Then we add a third characteristic emission of corpuscules with almost the velocity of light.

M. N. SAHA: If we ascribe to protons the velocity of about $60,000 \mathrm{~km} . / \mathrm{sec}$. the energy of the protons would be of the order of a million electron-volts.

\section{CONTRIBUTION TO THE DISCUSSION}

\section{BY K. O. KIEPENHEUER}

A flare is only part of a group of related phenomena, which all originate in one primary cause. This cause is obviously of magnetic nature and might be understood in terms of a magnetic disturbance rising from sub-photospheric layers into the chromosphere. According to our knowledge the following individual features have to be included:

Flares.

Activation of existing filaments.

Ejection of surges.

Line excitation in the chromosphere and the corona.

Emission of corpuscules with velocities from $3 \times 10^{7}$ to $6 \times 10^{9} \mathrm{~cm} . / \mathrm{sec}$.

Outbursts.

Emission of cosmic ray bursts. 
It seems reasonable to combine all these phenomena (which necessarily do not all occur together) into the term 'Solar Storm'. It will be difficult to understand a flare without handling it as a part of such a comprehensive solar event.

The following diagram represents a model of such a Solar Storm and its different components:

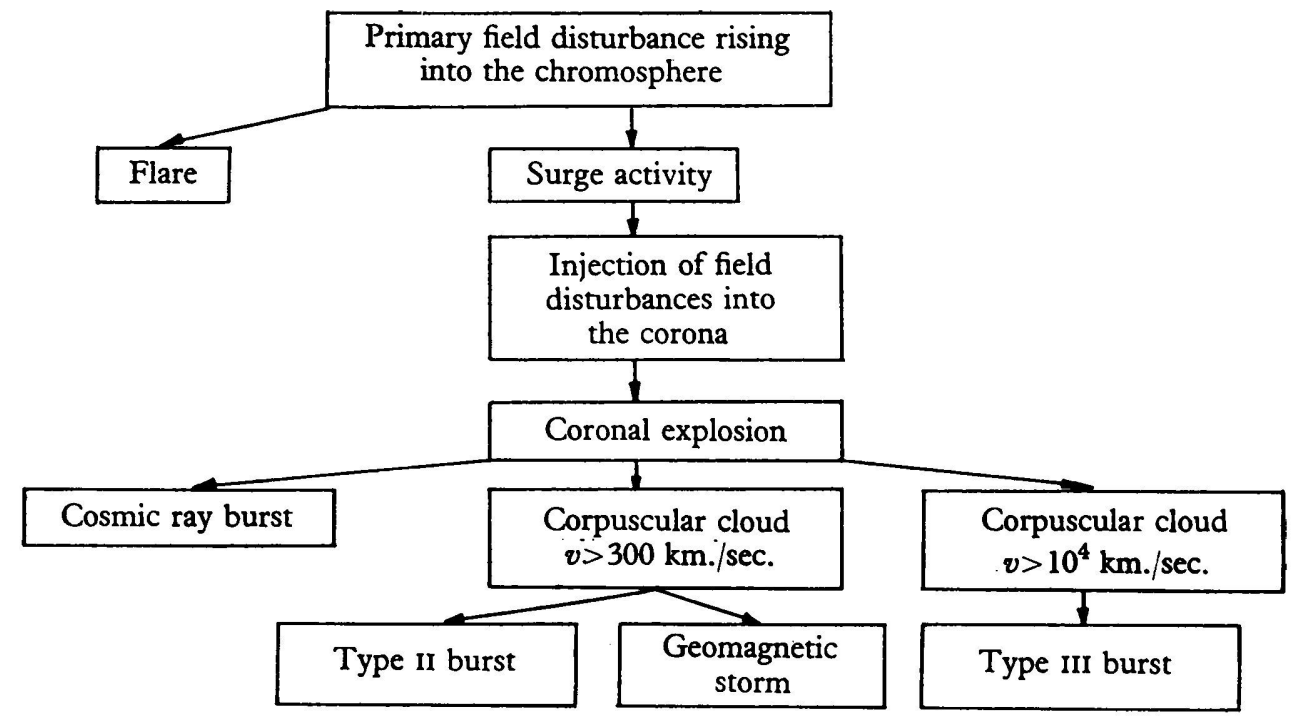

13. CONTRIBUTION TO THE DISCUSSION

By E. R. Mustel

I should like to make two remarks on the physics of solar flares. One very interesting problem is the origin of the bright continuous spectrum of a great chromospheric flare.

In a recent paper on this subject I considered two mechanisms. The first one is the recombination, connected with captures of electrons to different levels in the hydrogen atom. In certain cases $I$ found the energy emitted in this process to be sufficient to explain the continuous spectrum sometimes observed. However, this explanation meets the following difficulty: The mechanism leads to a very high intensity of the flares in the Lyman-continuum, in fact, too high to be consistent with the ionospheric observations. This problem requires further consideration.

The second explanation of the continuous spectrum seems to me the most probable one and can be described as follows: We know that the highly luminous matter of chromospheric flares occupies several levels in the solar atmosphere. Extreme cases are the photospheric $\mathrm{H} \alpha$ bombs first described by Ellerman and the flares appearing in coronal space. If we assume that the base of a very great chromospheric flare is situated somewhere in the photosphere, we may expect the temperature in this part of the photophere to increase during the flare. An increase in temperature of only $200^{\circ}$ is quite sufficient for explaining the observed continuous spectrum.

The other of my two remarks concerns the important problem involved in the excitation of atoms in solar flares. I shall consider here only the bright $\mathrm{H}$ and $\mathrm{K}$ lines of $\mathrm{Ca} \mathrm{II}$. In my papers on the physics of chromospheric faculae I have shown that the intensity of the ultra-violet doublet 3706 and 3737 of $\mathrm{Ca}$ II may be considered a sensitive criterion of the mechanism of excitation. If this mechanism is recombination, the $\mathrm{H}$ and $\mathrm{K}$ lines and the ultra-violet doublet will have comparable intensity. But if the mechanism is electronic impact, the intensity of the ultra-violet doublet must be much smaller than 